\title{
Tunable Nanoparticle Stability in Concentrated Polymer Solutions on the Basis of the Temperature Dependent Solvent Quality
}

Na Kyung Kwon", Chang Seo Park", Chae Han Lee", Yung Sam Kim, Charles F. Zukoski ${ }^{\S}$ and So Youn Kim *,\#

\section{Temperature Dependent Particle Stability with Varying Polymer Concentration}

We monitored particle stability at different polymer concentration $\left(R_{p}\right)$ and particle volume fractions $\left(\phi_{c}\right)$. While stable nanoparticles in PEG solutions look transparent blue, particles in gel look white opaque. As $R_{p}$ increases, rate of gelation decreases taking longer time to reach the gel state. When $R_{p}$ exceeds 0.5 , particles remain stable in a few months. As polymer concentration increases, solvent quality is less sensitive to the temperature and polymer correlation length is effectively decreased, which reduces depletion attractions. When $0.03<R_{p}<0.3$ and $\phi_{c} \geq 0.05$, the system becomes a gel with different rates. In Fig S1, we showed the gelation of nanoparticles at different $R_{p}$ and $\phi_{c}$.

\begin{tabular}{|c|c|c|c|c|c|c|c|c|c|c|c|c|}
\hline & & \multicolumn{5}{|c|}{$20^{\circ} \mathrm{C}$} & \multicolumn{6}{|c|}{$7^{\circ} \mathrm{C}$} \\
\hline & $R_{p}$ & 0.05 & 0.1 & 0.1 & 0.2 & 0.2 & $R_{p}$ & 0.05 & 0.1 & 0.1 & 0.2 & 0.2 \\
\hline & $\phi_{c}$ & 0.05 & 0.05 & 0.1 & 0.05 & 0.1 & $\phi_{c}$ & 0.05 & 0.05 & 0.1 & 0.05 & 0.1 \\
\hline 1 day & & & & & & & & & & & & \\
\hline 2 days & & & & & & & & & & & & \\
\hline 4 days & & & & & & & & & & & & \\
\hline 7 days & & & & & & & & & & & & \\
\hline
\end{tabular}

Figure S1. Temperature-dependent nanoparticle stability in polymer solutions at various conditions. 


\section{Turbidimetry}

The gelation of nanoparticles in polymer solution was studied with a Turbiscan online optical analyzer (Formulaction, France). In this device a near-infrared focused LED (wavelength $850 \mathrm{~nm}$ ) illuminates the scattering medium flowing through a cylindrical glass tube. Both the transmitted (detection angle $0^{\circ}$ ) and the backscattered (detection angle $135^{\circ}$ ) light flux are measured. Detailed information on this method is presented elsewhere. ${ }^{1}$

To probe the kinetic of particle aggregation, we have measured turbidity of the sample $\left(R_{p}=0.1\right.$, $\phi_{c}=0.1$ ) over time (every 0.5 hours, total 313 hours), scanning the sample in z-direction. Due to the instrumental limitation, temperature was kept at $60{ }^{\circ} \mathrm{C}$; gelation becomes slower than in $70{ }^{\circ} \mathrm{C}$, but the system became a gel eventually after 13 days. Figure S2 shows the scattered intensity in a transmission mode, where the scattered intensity decreases over time uniformly in the entire system from top to the bottom. Light scattering from nanoparticles in our system is considered to be Rayleigh scattering because the particles diameter, $d(40 \mathrm{~nm})$ is much smaller than incident light wavelength, $\lambda$ $(880 \mathrm{~nm}), d<<\lambda$. Thus, decreased scattered intensity in transmission mode directly indicates particles were being aggregated with time. Furthermore, the uniform decrease of intensity in $z$-direction confirms aggregation triggered uniform gelation throughout the system, rather than sedimentation.

In Fig S2, one can see the intensity decreases dramatically in the early stage of gelation, which indicates particles were aggregated presumably forming many clusters first and then, the aggregated particles create networks.

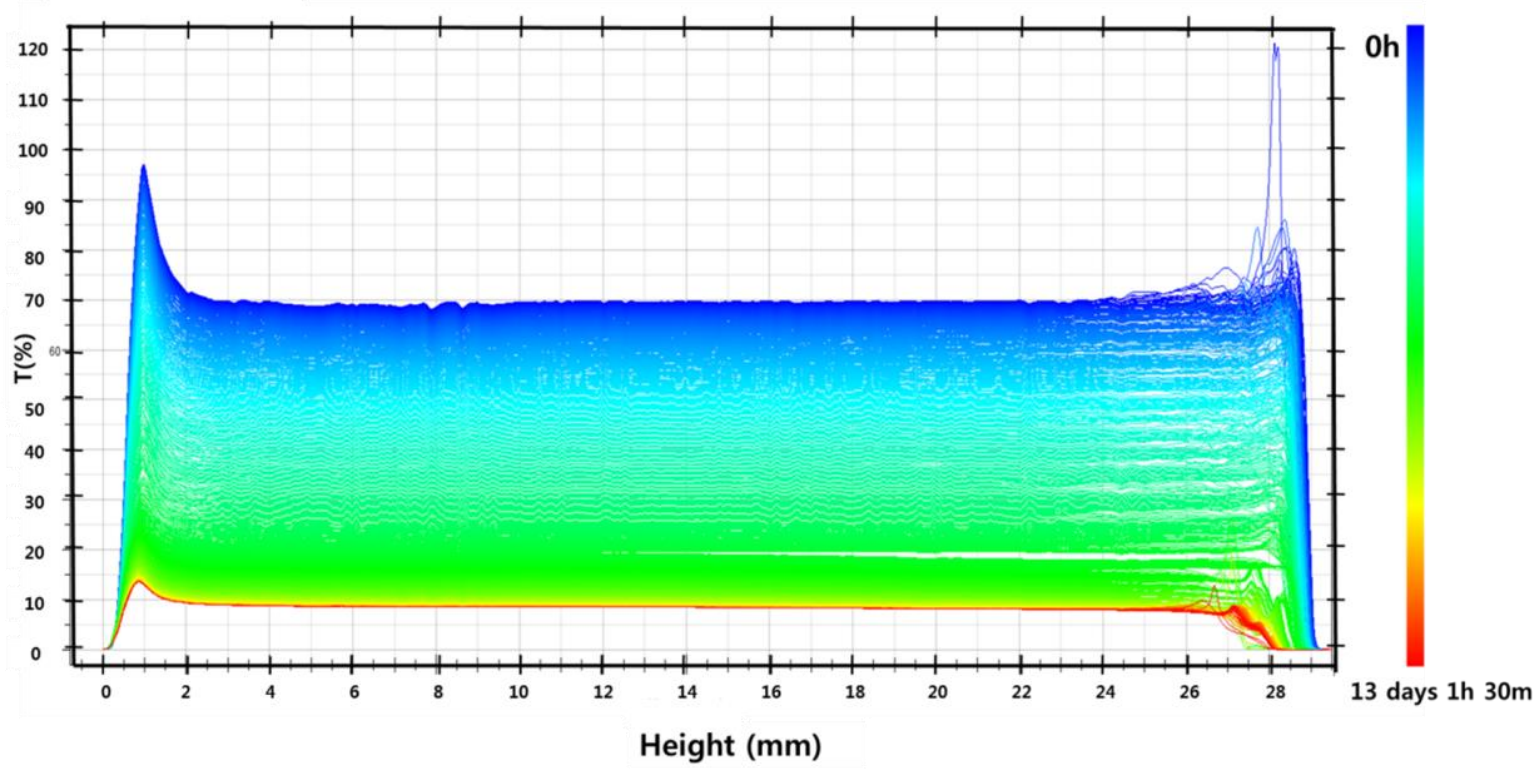

Figure S2. Change of turbidity for $R_{p}=0.1, \phi_{c}=0.1$ at $60{ }^{\circ} \mathrm{C}$ in transmission mode over time (13 days). $x$-axis indicates the sample height from the top (left) to the bottom (right) The partial increment of intensity at the top and bottom arisen from the surface meniscus effects. 


\section{Small-Angle X-ray Scattering (SAXS)}

We show the temperature-dependent particle stability at $R_{p}=0.5$ and $\phi_{c}=0.1$ below. While particle structure remains same at $20{ }^{\circ} \mathrm{C}$, it was significantly disrupted at $70{ }^{\circ} \mathrm{C}$ for 3 days. We did not observe any phase separation or gelation at this condition with the naked eyes.

(a)

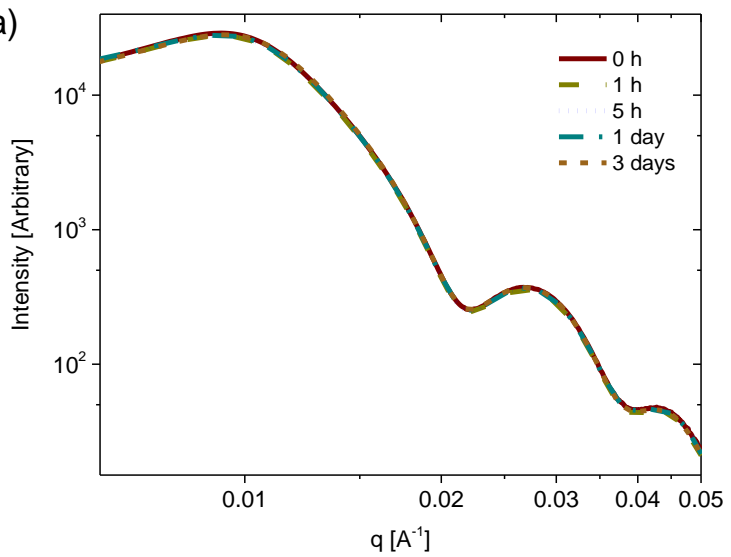

(b)

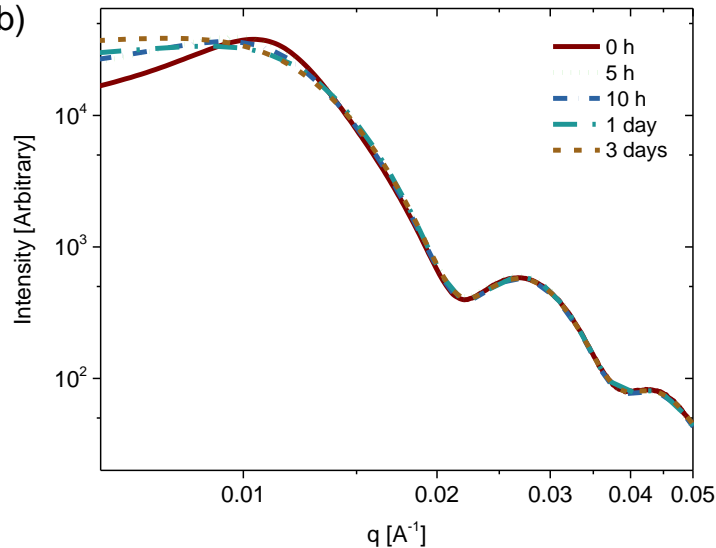

Figure S3. Scattered intensity profiles of the silica nanoparticles in PEG 400 suspensions with $R_{p}=$ 0.5 and $\phi_{c}=0.1$ (a) at $20{ }^{\circ} \mathrm{C}$ and (b) $70{ }^{\circ} \mathrm{C}$. 


\section{Zeta Potential Measurements}

(a)

Zeta Potential Distribution

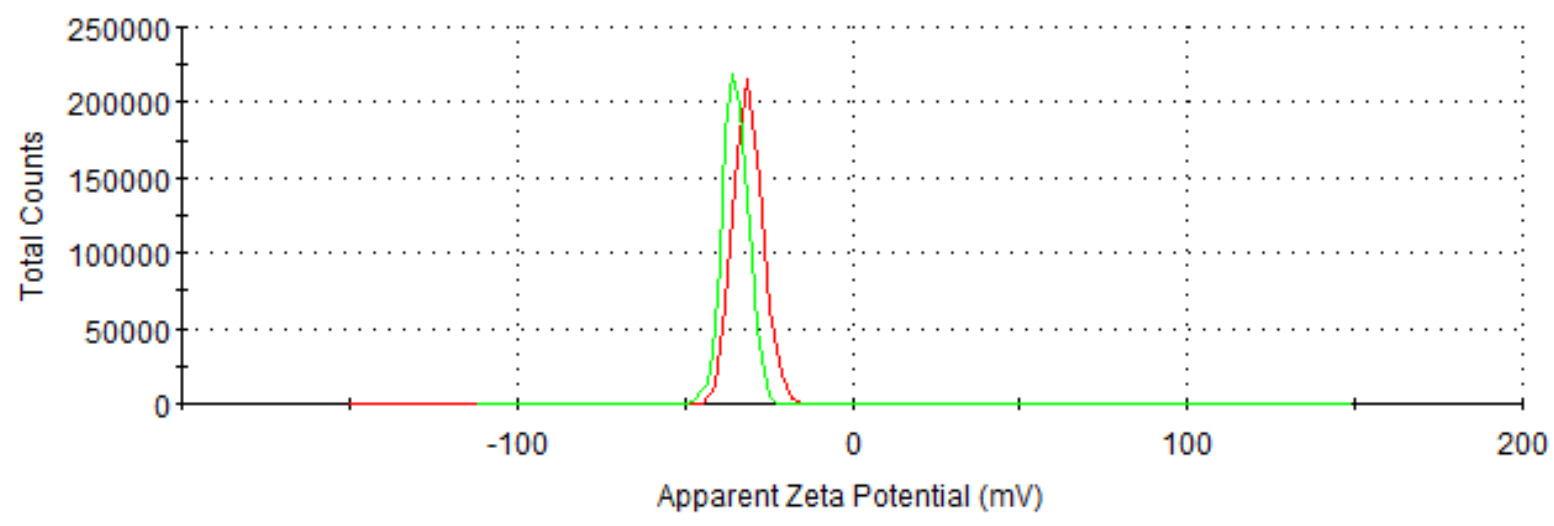

(b)

Zeta Potential Distribution

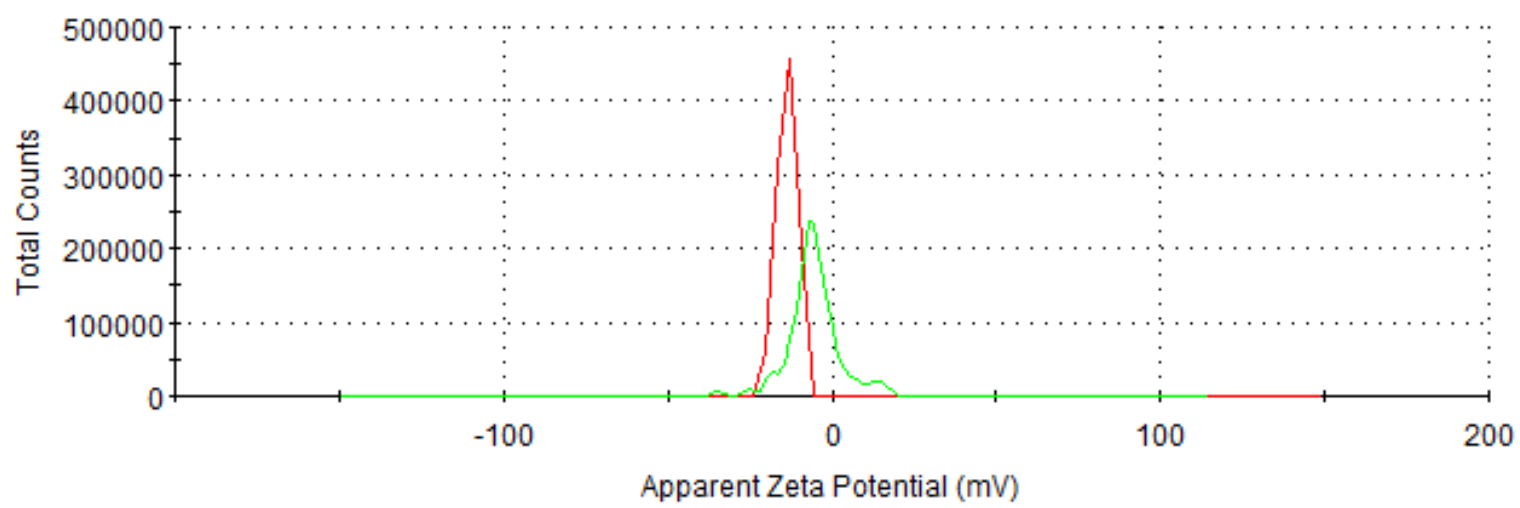

Figure S4. Zeta potential distribution of silica particles at $20{ }^{\circ} \mathrm{C}$ (red) and $70{ }^{\circ} \mathrm{C}$ (green) (a) in water and (b) in $R_{p}=0.1$ PEG solution. 


\section{Diffusing-Wave Spectroscopy (DWS)}

Particle Stability at Room Temperature. As mentioned in the main text, no observable change in the correlation function is found at $20{ }^{\circ} \mathrm{C}$ for 100 hours. Particles at this condition remain stable, showing liquid-like property over 9 months (Fig S5b).

The correlation functions below shows there was no significant change of structure over time. The relaxation time fluctuates a bit in the early stage and decreases. The initial fluctuation was originated from the sample preparation. To prepare the silica nanoparticles in polymer solution, $\left(\phi_{c}=0.1, R_{p}=\right.$ 0.1), a known amount of the PEG was added to the concentrated particle suspension and the solvent was quickly removed in a vacuum oven at $70{ }^{\circ} \mathrm{C}$, creating polymer-based nanocomposites. At this point, particle volume fraction $\left(\phi_{c}\right)$ is almost 0.55 , thus particles are closely packed before we add the deionized water. The polymer nanocomposites were then mixed with a known amount of deionized water in a vortex mixer, then, $\phi_{c}$ decreases to 0.10 .

Thus, particles have to reorganize the structures after adding solvent, requiring time for the dispersion to be homogeneous. Since particles were closely packed, it takes quite a time to reach in equilibrium. We confirmed the relaxation time and stretching factor are stabilized after 40 hours and no significant change is observed after that.
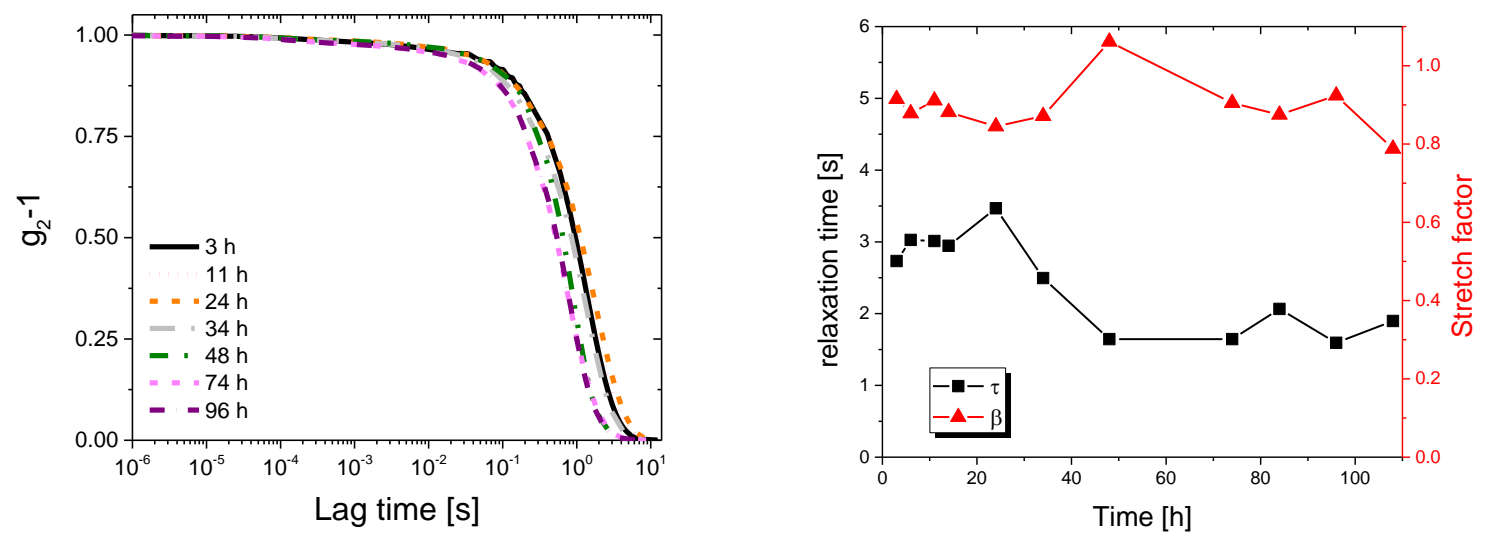

Figure S5a. (left) Time-resolved intensity autocorrelation functions of the silica nanoparticles and (right) results of fitting values for relaxation time and stretch factor of $\phi_{c}=0.1$, and $R_{p}=0.1$ at $20^{\circ} \mathrm{C}$. 

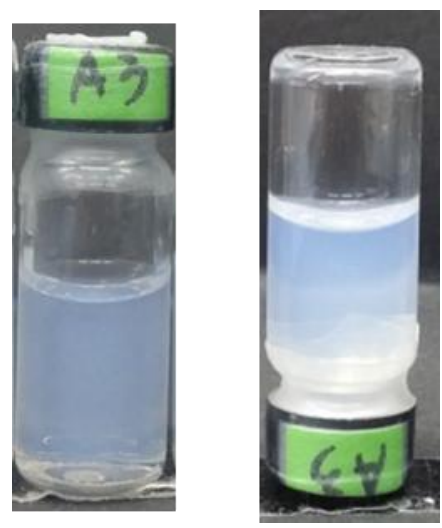

Figure S5b. Optical image for the sample ( $\left.\phi_{c}=0.1, R_{p}=0.1\right)$ stored at room temperature for 9 months.

The normalized intensity autocorrelation function $C(t)=g_{2}(t)-1$ was fitted with the equations in the main text. The below is an example of fitting quality after 2 hours at $70{ }^{\circ} \mathrm{C}$. The example was fitted with $f_{\text {long }}=0.9724, \tau_{\text {long }}=0.00819$ and $\tau_{\text {short }}=6.17 \times 10^{-5}$. Adjusted $\mathrm{R}^{2}$ was 0.9999 .

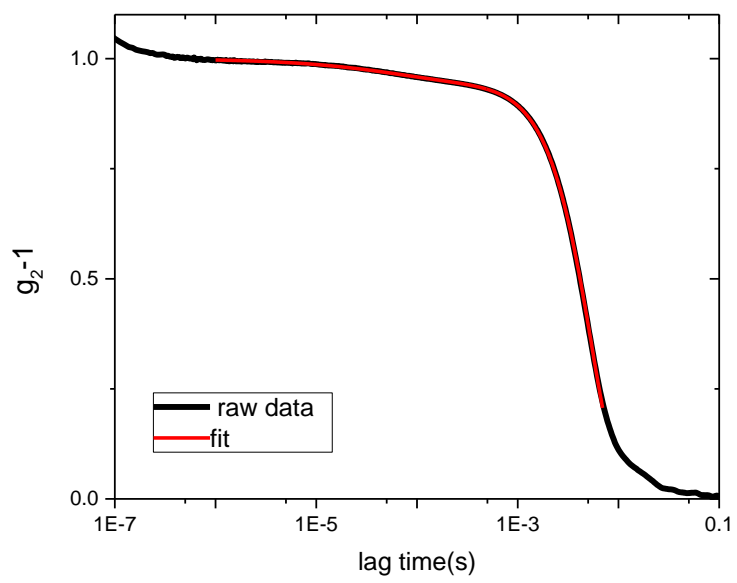

Figure S5c. Example of DWS fitting of $g_{2}(t)-1$. 


\section{Further Interpretation of Neutron Scattering Data in Fig. 3c and 3d}

The intensity, $I(q)$, of scattered neutrons at wave vector $q$, has three contributions:

$$
I(q)=n_{c} \Delta \rho_{c}^{2} P_{c}(q) S_{c c}\left(q, \phi_{c}\right)+2 \Delta \rho_{c} \Delta \rho_{p} \sqrt{n_{c} n_{p} P_{c}(q)} S_{c p}(q)+n_{p} \Delta \rho_{p}^{2} S_{p p}(q)
$$

where $n_{j}=\rho_{j}^{*} V_{j}^{2}$, and $\rho_{j}^{*}$ and $V_{j}$ are number density and unit volume of the $j^{\text {th }}$ component, respectively. $\Delta \rho_{j}$ is the difference of scattering length density of component $j$ and the medium, $P_{c}(q)$ is the particle form factor, and $S_{i j}(q)$ are the structure factors associated with two components $(p p, p c, c c)$ where the subscript $p(c)$ indicates polymer segments (particles). The details of how to extract partial structure factors were previously reported. ${ }^{2}$

From Fig. 3c in the main text, we could calculate the polymer-polymer structure factor shown in Fig. S6. The obtained $S_{p p}$ shows the existence of adsorbed polymer layers at $20{ }^{\circ} \mathrm{C}$.

Furthermore, we are able to extract the polymer shell form factor by having independent measures of $S_{c c}(q)$ determined where $\Delta \rho_{p}=0$ as: $P_{s}^{*}(q)=S_{p p} / S_{c c}$. This form factor can be fit with a core-shell model form factor using NCNR scattering fitting program ${ }^{3}$ :

$$
P_{s}^{*}(q)=\frac{C^{\prime}}{V_{\text {shell }}}\left[\frac{3 V_{c}\left(\rho_{c}-\rho_{\text {shell }}\right) j_{1}\left(q r_{c}\right)}{q r_{c}}+\frac{3 V_{\text {shell }}\left(\rho_{\text {shell }}-\rho_{s}\right) j_{1}\left(q r_{\text {shell }}\right)}{q r_{\text {shell }}}\right]^{2}
$$

Where $C^{\prime}$ is a constant, $j_{1}(x)=(\sin x-x \cos x) / x^{2}, r_{\text {shell }}=r_{c}+t, t$ is the layer thickness, $r_{c}$ is the radius of particle, $V_{i}=(4 \pi / 3) r_{i}^{3}$, and $\rho_{\text {shell }}$ is average value of the scattering length density within the adsorbed polymer layer. Particle polydispersity is taken into account, while the ratio $r_{c} / r_{\text {shell }}$ is held constant also using the NCNR curve fitting program. ${ }^{3}$ The detailed procedures are found in ref. ${ }^{2}$

The obtained polymer shell form factor is clearly differentiated from the single particle form factor as shown in Fig. S6.
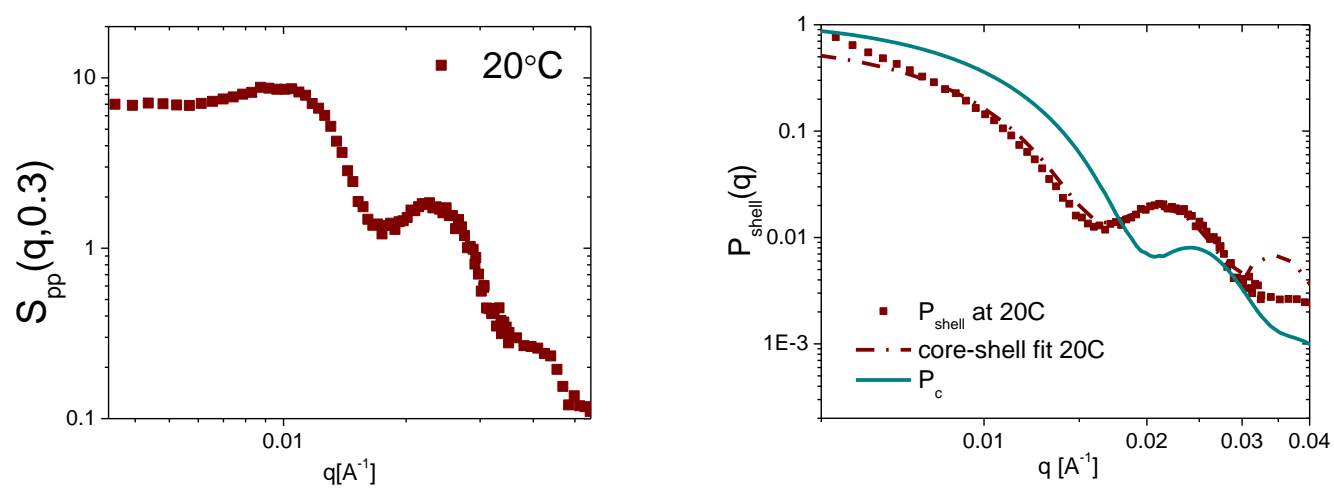
Figure S6. (left) $S_{p p}$ of polymer-polymer interactions obtained from Fig. 3c. (right) Form factor of adsorbed PEG layer in $20{ }^{\circ} \mathrm{C}$.

When temperature is raised to $70{ }^{\circ} \mathrm{C}$, the local concentration of bulk solution will be different as adsorbed polymers move. The case 1 is that polymers adsorb more strongly to the surface thus, local concentration $R_{p}$ in the bulk can be lower than 0.45 . (Note the initial bulk concentration is 0.45) Take an example of case 1 and suppose bulk $R_{p}$ is lowered to 0.37 . The calculated scattering length density of $R_{p}=0.37$ is shown in Fig. S7. Then, between the condition 'B' and 'F' the intensity from silica scattering can be increased back as $\Delta \rho_{\text {silica }}$ increases. However, we observed scatterings from silica nanoparticles continuously decrease from 'B' to ' $\mathrm{F}$ ' at $70{ }^{\circ} \mathrm{C}$ shown in Fig. $3 \mathrm{~d}$ in the main text, thus; this hypothesis is not correct.

The case 2 is that polymer moves to the bulk, thus bulk concentration would be slightly increased from 0.45 . Take an example of case 2 so set the bulk $R_{p}$ is 0.5 . Then, scattering from silica particles continuously decreases from ' $\mathrm{B}$ ' and ' $\mathrm{F}$ ' as we confirmed in Fig. $3 \mathrm{~d}$ in the main text. Furthermore, the match condition is no longer found at ' $\mathrm{F}$ ' noting $\Delta \rho_{\text {silica }}>0$ at $70{ }^{\circ} \mathrm{C}$ while $\Delta \rho_{\text {silica }} \sim$ 0 at $20{ }^{\circ} \mathrm{C}$.

To sum up, we could not observe the scatterings from adsorbed PEG layer at $70{ }^{\circ} \mathrm{C}$, ensuring polymer move to the bulk at elevated temperature.

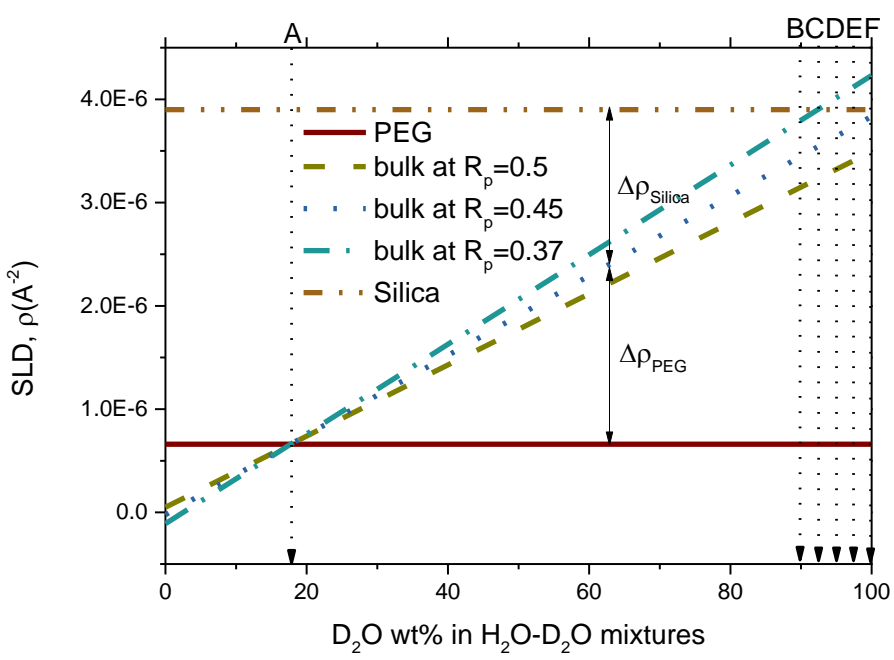

Figure S7. Scattering Length Density of silica nanoparticles, PEG and PEG solutions.

Since the adsorbed polymer chains at $20{ }^{\circ} \mathrm{C}$ moves to the bulk at $70{ }^{\circ} \mathrm{C}$, the SLD of dispersing phase also changes due to increased polymer concentrations, resulting in any $\mathrm{D}_{2} \mathrm{O} / \mathrm{H}_{2} \mathrm{O}$ ratio mixtures could not fully suppress scatterings from particles at $70{ }^{\circ} \mathrm{C}$. 


\section{FT-IR Spectra}

(a)

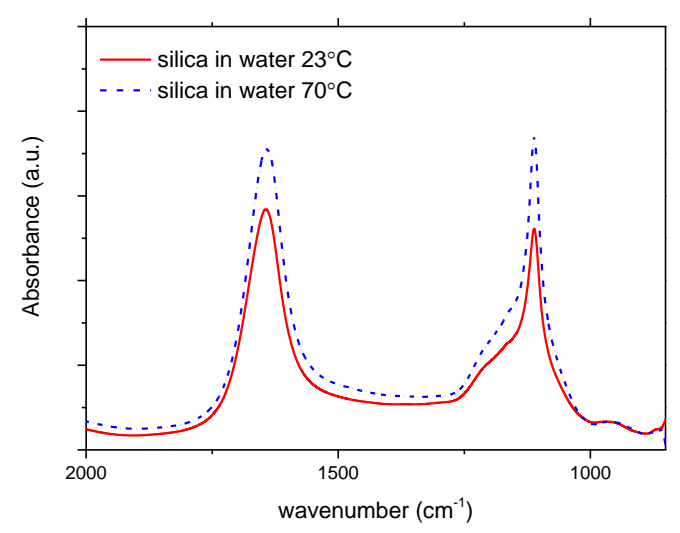

(b)

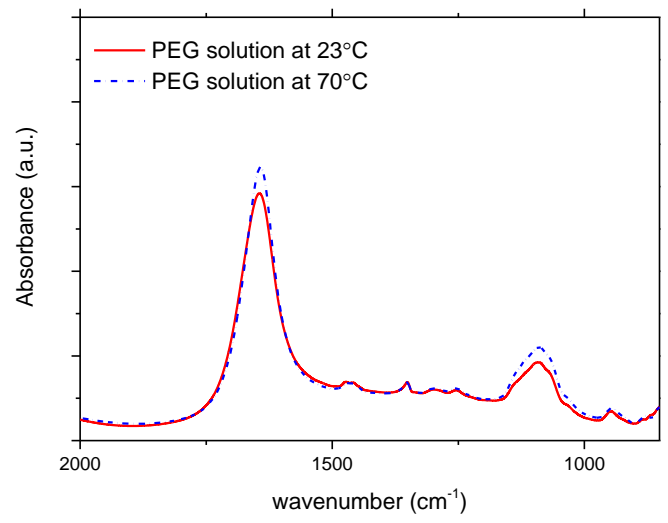

(c)

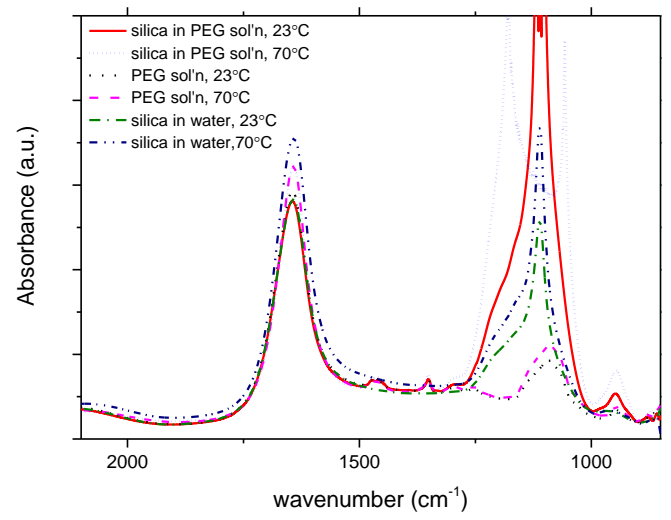

Figure S8. FT-IR spectra (a) for silica in water and (b) for PEG solutions at different temperatures. All FT-IR spectra is given in (c) for comparison. 


\section{Expected Total Pair Interaction Calculation}

The total interaction, $V(r)$, can be derived by Derjaguin, Landau, Verwey, and Overbeek (DLVO) theory ${ }^{4-5}$ with polymer-mediated interaction.

$$
V(r)=V_{A}(r)+V_{R}(r)+V_{P}(r)+V_{S}(r)
$$

DLVO theory considers van der Waals attractions, $V_{A}(r)$, and electrostatic repulsions, $V_{R}(r)$, while polymer mediated interaction includes depletion attractions, $V_{P}(r)$, and steric repulsion, $V_{S}(r)$.

Eq. (1) shows the summation of van der Waals attractions, electrostatic repulsions, depletion attractions, and steric repulsion.

\section{1) Van der Waals attractions}

The van der Waals attractions between a pair of particles can be approximated by Eq. (2) below. ${ }^{6}$

$$
V_{A}(r)=\left\{\begin{array}{ll}
\infty, & r<2 R+\delta_{a} \\
-\frac{A}{6}\left[\left(\frac{2 R^{2}}{r^{2}-4 R^{2}}\right)+\left(\frac{2 R^{2}}{r^{2}}\right)+\ln \left[\frac{r^{2}-4 R^{2}}{r^{2}}\right]\right], & r \geq 2 R+\delta_{a}
\end{array}\right\}
$$

where $A$ is Hamaker constant, $r$ is a center-to-center distance of particles, $R$ is the particle radius and $\delta_{a}$ is the nonzero distance of closest approach. Generally, the Hamaker constant is approximated by Eq. (3) for the symmetric case where two identical phases of 1 interact across continuous phase 3.

$$
A=\frac{3}{4} k T\left(\frac{\varepsilon_{1}-\varepsilon_{3}}{\varepsilon_{1}+\varepsilon_{3}}\right)^{2}+\frac{3 h v_{e}}{16 \sqrt{2}} \frac{\left(n_{1}^{2}-n_{3}^{2}\right)^{2}}{\left(n_{1}^{2}+n_{3}^{2}\right)^{3 / 2}}
$$

$v_{e}$ is the main electronic absorption frequency in the UV region, $3 \times 10^{-5} \mathrm{~s}^{-1}, h$ is Plank constant, $k$ is Boltzmann constant, $T$ is a temperature. $\varepsilon_{i}$ and $n_{i}$ are the relative dielectric constant and the refractive index of the $i$ th component, respectively. In this study, the dispersing phase 1 is the silica nanoparticles and the continuous phase 3 is a homogeneous mixture of PEG400 and water. Based on the volume fraction of polymers in the solution, $\varepsilon_{i}$ and $n_{i}$ are adjusted.

$$
\begin{aligned}
& \varepsilon_{3}\left(T, R_{p}\right)=\varepsilon_{P E G}(T) R_{p}+\varepsilon_{\text {water }}(T)\left(1-R_{p}\right) \\
& n_{3}\left(T, R_{p}\right)=n_{P E G}(T) R_{p}+n_{\text {water }}(T)\left(1-R_{p}\right)
\end{aligned}
$$

where $R_{p}$ is the volume fraction of PEG 400 in the continuous phase. ${ }^{7}$ Because $\varepsilon_{i}$ and $n_{i}$ are dependent on temperature, $\varepsilon_{3}$ and $n_{3}$ are calculated accordingly. For silica in $R_{p}=0.1$ solution, $\varepsilon_{\text {silica }}=3.8, \varepsilon_{P E G 400}$ $=12.5, \varepsilon_{\text {water }}=80.1, n_{\text {silica }}=1.4584, n_{P E G 400}=1.14667$, and $n_{\text {water }}=1.3332$ were used at $20{ }^{\circ} \mathrm{C}$ where $\varepsilon_{\text {silica }}=3.8, \varepsilon_{P E G 400}=10, \varepsilon_{\text {water }}=63.857, n_{\text {silica }}=1.4589, n_{P E G 400}=1.44904$, and $n_{\text {water }}=1.3250$ were used at $70{ }^{\circ} \mathrm{C}^{8-12}$

Through the Eq. (3), the Hamaker constant was $-1.431 k T$ and $-1.407 k T$ at $20{ }^{\circ} \mathrm{C}$ and $70{ }^{\circ} \mathrm{C}$, 
respectively.

\section{2) Electrostatic repulsions}

Silica nanoparticles are negatively charged by the dissociation of silanol group. Previous study reported silica nanoparticles are highly charged in water or ethanol, thus remain stable for a long time. ${ }^{13}$ In the presence of polymers, however, surface charged can be effectively dropped because electron permittivity is lowered and polymers may promote the deionization of $\mathrm{SiO}^{-}$groups as we mentioned in the main text.

The repulsive pair potential can be written, ${ }^{14}$

$$
V_{R}(r)=4 \pi \varepsilon_{r} \varepsilon_{0} R^{2} q_{0}^{2} \exp (2 R \kappa) \frac{\exp [-\kappa r]}{r}
$$

where $\varepsilon_{0}$ is the permittivity of vacuum, $\varepsilon_{r}$ is the relative permittivity of continuous phase, $q_{0}$ is the zeta potential on the surface of the particles and $\kappa$ is the Debye-Hückel parameter whose inverse term is the charge screening length of particles. $q_{0}$ and $\kappa$ are estimated from zeta potential measurement of nanoparticles and conductivity measurement of the continuous phase.

$$
\sigma=\sigma_{0}+\frac{12 \varepsilon_{r} \varepsilon_{0}}{D^{2} \exp [-\kappa D / 2]}\left[z_{i} w_{i} \zeta+\frac{2 \varepsilon_{r} \varepsilon_{0}}{3 \mu} \zeta^{2}\right] \phi_{c}
$$

where $\sigma$ is the solution conductivity, $\sigma_{0}$ is the conductivity of the continuous phase, $D$ is the particle diameter, $z_{i}$ is the valence of the counterion, $w_{i}$ is the mobility of the $i$ th ion in continuous phase, $\mu$ is the viscosity of the solution and $\phi_{c}$ is the particle volume fraction. At $20^{\circ} \mathrm{C}, \sigma_{0}=0.0705 \mathrm{mS} / \mathrm{cm}, \sigma=$ $0.146 \mathrm{mS} / \mathrm{cm}, \zeta=-13.7 \mathrm{mV}, w_{i}=-0.9267 \times 10^{-8} \mathrm{~m}^{2} / \mathrm{V} \cdot \mathrm{s}$, and $\mu=14.62 \mathrm{mPa} \cdot \mathrm{s}$. At $70{ }^{\circ} \mathrm{C}, \zeta=-5.74 \mathrm{mV}$. From this parameters with Eq.(6), we obtained $\kappa=0.245 \mathrm{~nm}^{-1}$.

\section{3) Depletion attractions}

Although PEGs are known to be strongly adsorbed on the silica surface, the remaining polymers in bulk create depletion attractions. To estimate the depletion attractions, polymer reference interaction site model (PRISM) integral equation-based theory was employed in this system. The details for the theory developments and its validations are elsewhere. ${ }^{15}$

Based on this theory, we approximate the depletion attraction by $W_{p}(r)$, the polymer-mediated contribution to the particle-particle correlation function based on athermal PY closure,

$$
\begin{gathered}
V_{P}(r)=-k T \log \left(1+W_{P}(r)\right) \\
W_{P}(r)=\frac{\pi z}{3}\left(\frac{R}{r}\right)\left(\frac{R}{d}\right) e^{-(r-2 R) / \xi_{P}}, r>2 R
\end{gathered}
$$




$$
\frac{d}{\xi_{P}}=\frac{d}{\xi_{C}}+\frac{\pi z}{3} ; \quad \frac{d}{\xi_{C}}=\sqrt{\frac{12}{N}} ; \quad R_{g}=d \sqrt{\frac{N}{6}} \quad z=\rho_{p} d^{3}=\left(\frac{N N_{A} c_{P}}{M w_{P}}\right) d^{3} \quad \ldots
$$

Where $\rho_{p}$ is the polymer segment number density, $d$ is the effective statistical segment length, $N$ is a degree of the polymerization, $N_{A}$ is Avogadro's number, $c_{P}$ is the polymer concentration $(\mathrm{g} / \mathrm{mL})$, and $M w_{P}$ is the molecular weight of the polymer. In this approach, the two screening lengths are introduced, one is the polymer density screening length, $\xi_{P}$, and the other is the colloid density screening length, $\xi_{C}$. The calculation result is shown in the Fig. 5 in the main text.

\section{4) Steric repulsion}

The adsorbed polymer layers on silica nanoparticles create steric layer preventing the approach of other particles. Since our proposed mechanism concludes the loss of steric layers is attributed to the particle aggregation, the estimation of steric repulsion can be critical.

Many previous studies quantified the amount of PEGs onto silica particles and several models have been suggested to estimate the steric repulsion. ${ }^{16-19}$ Kim et al. ${ }^{13}$ showed the similarity of repulsive potentials from de Gennes's scaling theory ${ }^{17}$ and Mackor's theory. ${ }^{18-19}$ In our current experimental system, we quantified steric repulsion from the model by Mackor. ${ }^{13,18-20}$ Polymers are treated as rigid rods whose one ends are anchored to the surface of the particles and the other is free from the particles, thus can interact within the bulk polymers. This model estimates the reduction of the entropy, resulting from restriction of segment movement by opposing surface. Thus, this steric effect can be written,

$$
V_{S}=\left\{\begin{array}{ll}
N_{S} k_{B} T \theta_{\infty} \pi(\delta-x)^{2}\left(\frac{2 R+\delta+x}{\delta}\right), & \text { for } \mathrm{x}<\delta \\
0, & \text { for } \mathrm{x}<\delta
\end{array}\right\}
$$

Where $N_{S}$ is the number of adsorbed sites per unit area, $\theta_{\infty}$ is the surface coverage $(\sim 0.2$ for polymer adsorption), and $x$ is the surface to surface separated distance between particles. The key parameter is $N_{S}$, which is estimated $N_{S}=2.3 \times 10^{18} \mathrm{~m}^{-2}$, with $\theta_{\infty}=0.19$ from the adsorption isotherm data, shown in Trens et al. ${ }^{16}$ However, we should note that the exact determination of $N_{S}$ can be complicated, thus only an approximation. The adsorbed layer thickness $\delta$ is in the same order of $R_{g}$ of PEG 400, thus we set $\delta \sim 0.84 \mathrm{~nm}$. Based on the Eq. (10), the steric layer potentials at contact is around 20-30 kT.

Through the calculation, we extracted and plotted the total pair interaction potentials for each term and its summation in Fig.5. As predicted, the existence of steric effect gives a major contribution to the particle stability. Therefore, gelation from particle aggregations is attributed to the loss of steric layer. 
References

1. Mengual, O.; Meunier, G.; Cayre, I.; Puech, K.; Snabre, P., Characterisation of instability of concentrated dispersions by a new optical analyser: the TURBISCAN MA 1000. Colloid. Surface. A. 1999,152 (1-2), 111-123.

2. Kim, S. Y.; Schweizer, K. S.; Zukoski, C. F., Multiscale Structure, Interfacial Cohesion, Adsorbed Layers, and Thermodynamics in Dense Polymer-Nanoparticle Mixtures. Phys. Rev. Lett. 2011,107 (22).

3. Kline, S. R., Reduction and analysis of SANS and USANS data using IGOR Pro. J. Appl. Crystallogr. 2006,39, 895-900.

4. Derjaguin, B. V. L., L., Theory of the Stability of Strongly Charged Lyophobic Sols and of the Adhesion of Strongly Charged Particles in Solutions of Electrolytes. Acta Physicochim. URSS 1941, 14, 633-662.

5. Verwey, E. J. W. O., J. T. G., Theory of the Stability of Lyophobic Colloids. Elsevier: Amsterdam 1948.

6. Israelachvili, J. N., Intermolecular and Surface Forces. 2 ed.; Academic press: New York, 1991.

7. Reis, J. C. R.; Iglesias, T. P.; Douheret, G.; Davis, M. I., The Permittivity of Thermodynamically Ideal Liquid Mixtures and the Excess Relative Permittivity of Binary Dielectrics. Phys. Chem. Chem. Phys. 2009,11 (20), 3977-3986.

8. I. Thormählen, J. S., and U. Grigull, Refractive Index of Water and Its Dependence on Wavelength, Temperature, and Density. J. Phys. Chem. Ref. Data 1985,14, 933.

9. Leviton, D. B.; Frey, B. J., Temperature-dependent Absolute Refractive Index Measurements of Synthetic Fused Silica - art. no. 62732K. In Optomechanical Technologies for Astronomy, Pts 1 and 2, AtadEttedgul, E.; Antebi, J.; Lemke, D., Eds. Spie-Int Soc Optical Engineering: Bellingham, 2006; Vol. 6273, pp K2732-K2732.

10. Zivkovic, N.; Serbanovic, S.; Kijevcanin, M.; Zivkovic, E., Volumetric Properties, Viscosities, and Refractive Indices of the Binary Systems 1-Butanol + PEG 200, + PEG 400, and + TEGDME. Int. J. Thermophys. 2013,34 (6), 1002-1020.

11. Koizumi, N.; Hanai, T., Dielectric Propeties of Lower-membered Polyethylene Glycol at Low Frequencies. J. Phys. Chem. 1956,60 (11), 1496-1500.

12. Malmberg, C. G.; Maryott, A. A., Dielectric Constant of Water from $0^{\circ}$ to $100^{\circ} \mathrm{C}$. J. Res. Nat. Bur. Stand. 1956,56 (1), 1-8.

13. Kim, S. Y.; Zukoski, C. F., Particle Restabilization in Silica/PEG/Ethanol Suspensions: How Strongly do Polymers Need To Adsorb To Stabilize Against Aggregation? Langmuir 2011,27 (9), 5211-5221.

14. Russel, W. B., D. A. Saville, and W. R. Schowalter, Colloidal Dispersions. Cambridge University Press: Cambridge, 1989.

15. Chatterjee, A. P.; Schweizer, K. S., Influence of Solvent Quality and Thermal Fluctuations on Polymer-mediated Depletion Interactions. Macromolecules 1999,32 (3), 923-934.

16. Trens, P.; Denoyel, R., Conformation of Poly(ethylene glycol) Polymers at the Silica Water Interface - A Microcalorimetric study. Langmuir 1993, 9 (2), 519-522.

17. De Gennes, P. G., Polymer-Solutions Near an Interface.1. Adsorption and Depletion Layers. Macromolecules 1981,14 (6), 1637-1644.

18. Mackor, E. L., A Theoretical Approach of the Colloid-Chemical Stability of Dispersion in Hydrocarbons. J. Colloid. Interf. Sci 1951,6 (5), 492-495.

19. Mackor, E. L.; Vanderwaals, J. H., The Statistics of the Adsorption of Rod-Shaped Molecules in Connection with the Stability of Certain Colloidal Dispersions. J. Colloid. Interf. Sci 1952,7 (5), 535-550.

20. Sato, T., Adsorption of Polyamides and the Stability of Dispersion. J. Appl. Polym. Sci. 1971,15 (5), 1053-1067. 
